\title{
A three-dimensional anatomy of the posterolateral compartment of the knee: the use of a new technology in the study of musculoskeletal anatomy
}

\author{
This article was published in the following Dove Press journal: \\ Open Access Journal of Sports Medicine \\ 20 January 2012 \\ Number of times this article has been viewed
}

\author{
Diego Costa Astur' \\ Gustavo Gonçalves Arliani' \\ Camila Cohen Kaleka ${ }^{2}$ \\ Wahy Jalikjian ${ }^{3}$ \\ Pau Golano ${ }^{4,5}$ \\ Moises Cohen'
}

'Department of Orthopedics and Traumatology, Universidade Federal de São Paulo (UNIFESP), São Paulo, ${ }^{2}$ Department of Orthopedics and Traumatology, Faculdade de Medicina da Santa Casa de Misericórdia de São Paulo, São Paulo, ${ }^{3}$ Department of Orthopedics and Traumatology, Faculdade de Medicina de São José do Rio Preto, São José do Rio Preto, Brazil; ${ }^{4}$ Laboratory of Arthroscopic and Surgical Anatomy, Department of Pathology and Experimental Therapeutics (Human Anatomy Unit), University of Barcelona, Barcelona, Spain; ${ }^{5}$ Orthopedic Surgery Department, Pittburgh University, Pittsburgh, PA, USA

\author{
Correspondence: Diego Costa Astur \\ Departamento de Ortopedia \\ e Traumatologia da Universidade \\ Federal de São Paulo, Rua Borges Lagoa, \\ 783, $5^{\circ}$ andar, Vila Clementino, \\ São Paulo CEP 04038-032, Brazil \\ Email mcastur@yahoo.com
}

Background: Recently, an interest has developed in understanding the anatomy of the posterior and posterolateral knee. The posterolateral compartment of the knee corresponds to a complex arrangement of ligaments and myotendinous structures. Undiagnosed lesions in this compartment are the main reason for failure of the anterior and posterior cruciate ligament reconstructions. Understanding the anatomy of these structures is essential to assist in the diagnosis and treatment of these lesions. The aim of this study was to better understand the relationship between these structures of the knee using three-dimensional technology.

Methods: Ten knees were included from cadaver lower limbs of adult patients. The skin and subcutaneous tissue were removed leaving only the muscle groups and ligaments. The neurovascular bundles and their ramifications were preserved. Images were acquired from the dissections using a Nikon D40 camera with AF-S Nikkor 18-55 mm (1:3.5 5.6 GII ED) and Micro Nikkor $105 \mathrm{~mm}(1: 2.8)$ lenses. The pair of images were processed using Callipyan 3D and AnaBuilder software, which transforms the two images into one anaglyphic image.

Results: During the dissection of the knees, twelve pictures were acquired and transformed into anaglyphic images.

Conclusion: The use of three-dimensional images in this study demonstrates that this technique is useful to improve the knowledge in anatomy of the knee as well as for knee reconstruction surgery.

Keywords: knee joint anatomy and histology, humans, anatomy education, photography methods, education, medical methods, eyeglasses

\section{Introduction}

Macroscopic and microscopic studies of the anatomy of the knee have resulted in a better understanding of joint biomechanics. ${ }^{1,2}$ Novel techniques for the treatment of ligament injuries are emerging with the purpose of performing an anatomical reconstruction to improve both short-term and long-term function. ${ }^{3,4}$

An interest has developed in understanding the anatomy of the posterior and posterolateral compartment of the knee in the last few years. The posterolateral compartment of the knee corresponds to a complex arrangement of ligaments and myotendinous structures. The primary function of the posterolateral corner of the knee is to resist the forces of adduction, external rotation, and a posterior tibial translation. Injuries to these structures can cause instability and disability that can compromise daily activities. ${ }^{5}$ Undiagnosed posterolateral corner instability can be a reason for failure submit your manuscript | www.dovepress.com

Dovepress

http://dx.doi.org// 0.2147/OAJSM.S28705
Open Access Journal of Sports Medicine 2012:3 I-5

(C) 2012 Astur et al, publisher and licensee Dove Medical Press Ltd. This is an Open Access article which permits unrestricted noncommercial use, provided the original work is properly cited. 
in anterior and posterior cruciate ligament reconstruction. ${ }^{6,7}$ Understanding the anatomy is essential in the diagnosis and treatment of injuries to these structures. ${ }^{5}$

The aim of this study was to use three-dimensional (3D) technology to visualize the anatomical features and relationship of the structures of the posterolateral corner of the knee, with a particular attention to the vessels of this compartment, which are not so well described in the literature.

\section{Methods}

This research project was evaluated and approved by the Ethics Committee. This study comprised of ten knees from cadaver lower limbs of adult patients aged between 34 and 55 years (average: 41.2 years). The dissected knees were from seven men and three women. Skin and subcutaneous tissues were removed exposing the muscle groups and ligaments. The neurovascular bundles and their ramifications were preserved.

A silicone rubber with red pigment was injected into the popliteal artery for a better visualization of the popliteal branches making it possible to study all of the knee vascular anatomy.

Images were acquired from the dissections using a Nikon D40 camera with AF-S Nikkor 18-55 mm (1:3.5 5.6 GII ED) and Micro Nikkor $105 \mathrm{~mm}$ (1:2.8) lenses (Nikon Corporation, Tokyo, Japan). These lenses were placed on a slide bar attached to a tripod. The slider is used to generate two images of the same structure, which differ in the horizontal angle proportional to the interpupillary distance. ${ }^{8-11}$ The pair of images were processed using Callipyan 3D and AnaBuilder software, which transforms the two images into one anaglyphic image. Special anaglyph 3D glasses enable visualization of the $3 \mathrm{D}$ images.

\section{Results}

The first images of the lateral compartment indicate the appearance of structures usually visualized during surgery including the iliotibial band, biceps femoris, and lateral head of the gastrocnemius muscles, and their relationship to the fibular nerve. These structures are considered the first layer of the posterolateral compartment (Figure 1).

The iliotibial band, the biceps, and the lateral head of the gastrocnemius are reflected while maintaining their proximal femoral insertion. At this time, it was possible to more precisely visualize the proximal and distal limits of the lateral collateral ligament, the popliteus muscle, and the popliteofibular ligament. These three structures are considered the second layer of the posterolateral compartment (Figures 2-4).

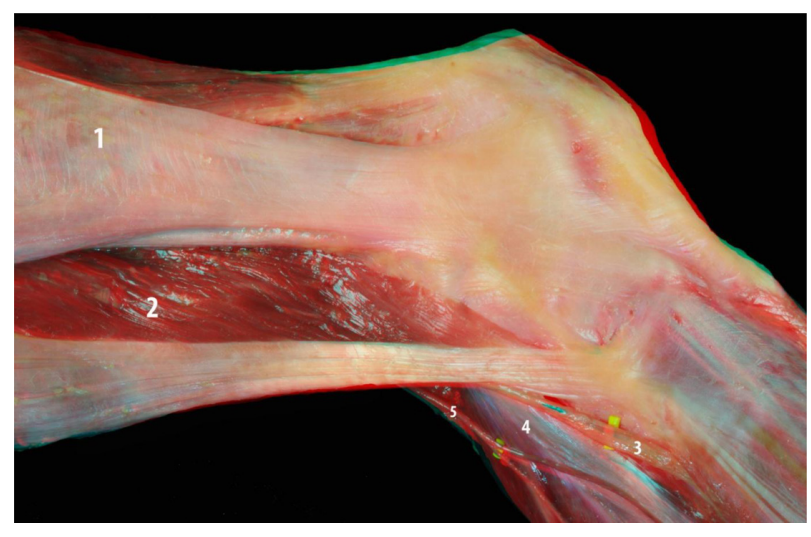

Figure I Flexed knee: iliotibial band (I), biceps femoris (2), fibular nerve (3), lateral gastrocnemius tendon (4), and sural nerve and ramifications (5).

It was possible to visualize the vascularity of the knee, especially the posterolateral corner and lateral side, using a contrast injection of a mix of silicone and red dye (Figures 5 and 6).

The lateral inferior genicular artery helps delineate the popliteus tendon and popliteofibular ligament. Its path is superficial to the popliteus tendon and the popliteofibular ligament, and it is deep to the collateral lateral ligament, bordering the anterior horn of the lateral meniscus (Figure 7). The lateral superior genicular artery branches are concentrated in the lateral femoral condyle (Figure 8).

\section{Discussion}

The use of 3D technology to visualize anatomic structures may provide a better understanding of the structures and their relationships to each other. The use of 3D imaging is already frequently in the entertainment industry and a technical note about the use of 3D musculosqueletal anatomy and neurosurgery anatomy studies has been published previously. ${ }^{8,12}$

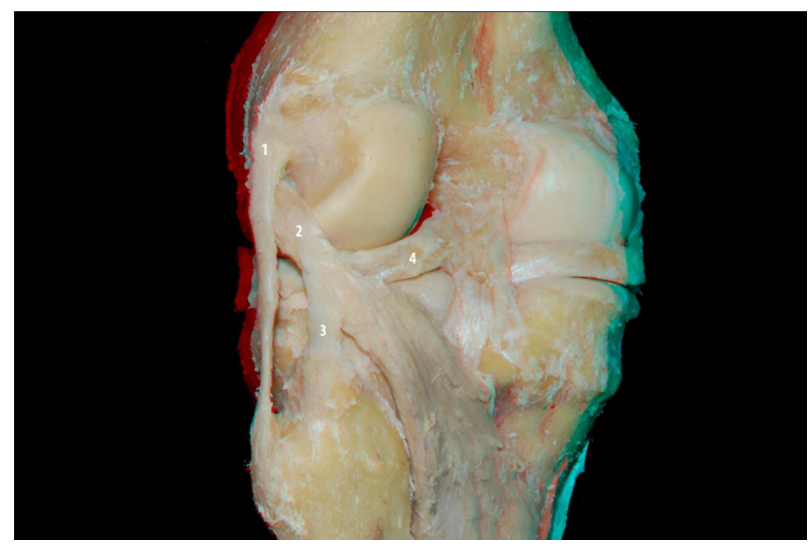

Figure 2 Posterior view of the knee: lateral collateral ligament (I), popliteus tendon (2), popliteofibular ligament (3), and lateral meniscus (4). 


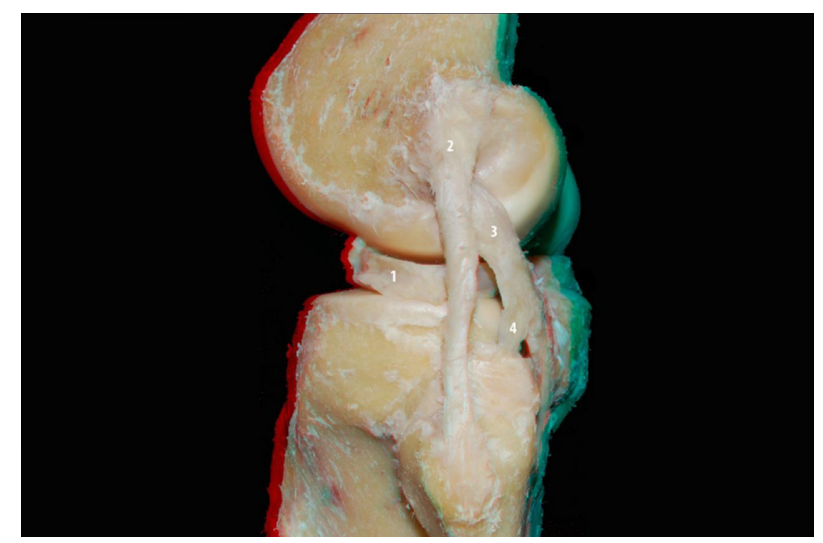

Figure 3 Lateral view of the posterolateral compartment of the extended knee: lateral meniscus (I), lateral collateral ligament (2), popliteus tendon (3), and popliteofibular ligament (4).

This study demonstrates that it can be applied to the structures of the knee and helps provide further insight to these areas.

Clinicians who are less familiar with this study area, such as residents and even orthopedic knee surgeons, will be able to use this tool to more easily understand and visualize these structures. For example, it is easier to see an artery interacting with a tendon and penetrating a ligament using 3D technology than using a single plane, eg, the lateral branches of the popliteal artery: the inferior and superior lateral genicular artery. With 3D image studies people are able to understand relationships of these vessels that were not elucidated before.

The lateral inferior genicular artery borders the lateral meniscus and is responsible for its blood supply. It ends in the femoral intercondylar, being responsible for part of the irrigation of the central ligaments of the knee: anterior cruciate ligament and posterior cruciate ligament (Figure 9). During its course it is clear that the interaction between this artery

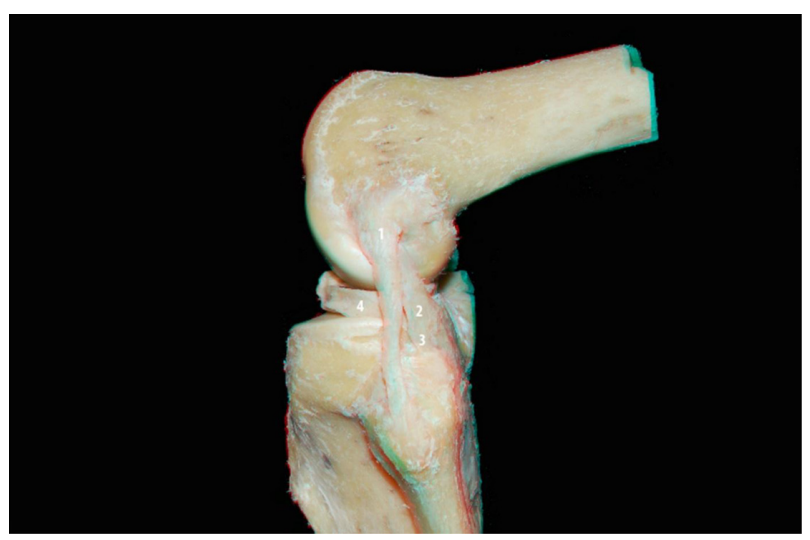

Figure 4 Lateral view of the posterolateral compartment of the flexed knee: lateral collateral ligament (I), popliteus tendon (2), popliteofibular ligament (3), and lateral meniscus (4).

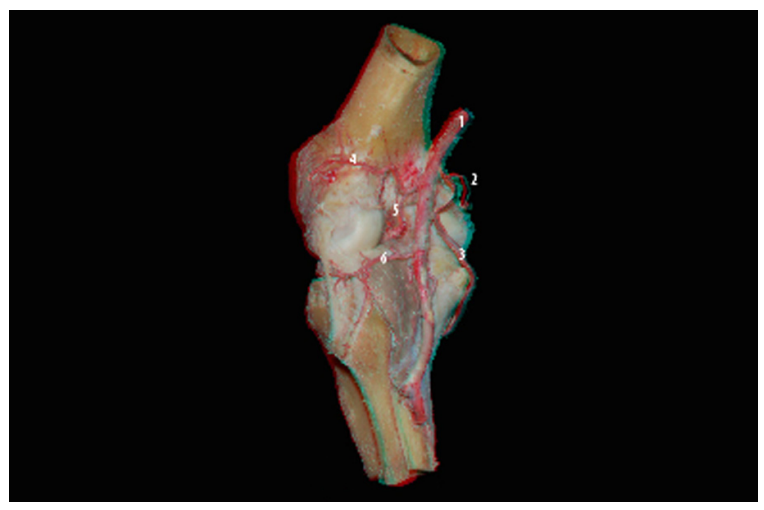

Figure 5 Posterior view of the knee: popliteal artery ( 1 ) and ramifications: medial superior genicular artery (2), medial inferior genicular artery (3), lateral superior genicular artery (4), middle genicular artery (5), and lateral inferior genicular artery (6)

and the fat body of the lateral meniscus is quite complex, invalidating the anatomical dissection between them. During a meniscus suture or meniscus repair for the reconstruction of a lateral plateau fracture some of these vessels, or even the main branch of the inferior lateral genicular artery, may be ripped. It is unusual to see this detail in $2 \mathrm{D}$ images or even during the surgical procedure. As it is not always possible for surgeons in the beginning of their careers to gain experience on less common surgeries, such as reconstruction of the posterolateral compartment of the knee, this technology enables them to familiarize themselves with the anatomy of the knee using 3D images.

The lateral superior genicular artery branches are concentrated in the lateral femoral condyle. It is mainly responsible for the vascularization of all this condyle with a smaller artery called lateral supracondylar artery. The large number of branches that penetrate the subchondral bone around the lateral condyle may explain the lower incidence

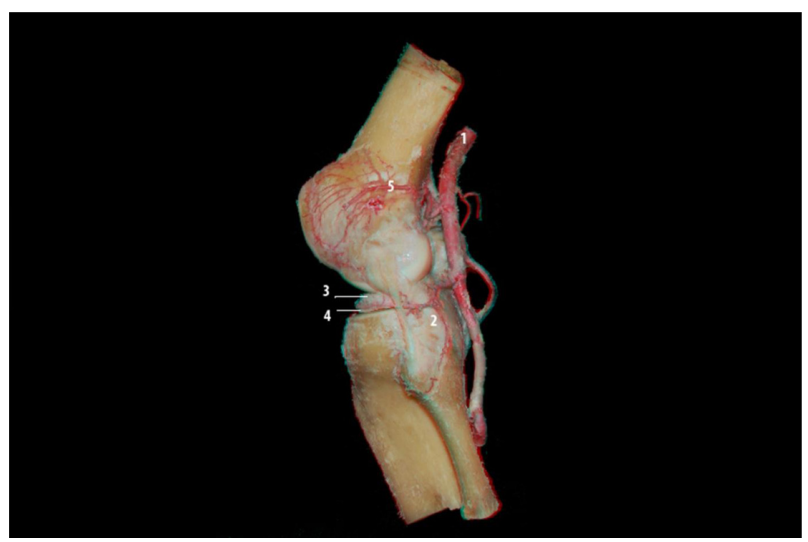

Figure 6 Lateral view of the knee: popliteal artery (I), popliteofibular ligament (2), lateral meniscus (3), lateral inferior genicular artery (4), and lateral superior genicular artery (5). 


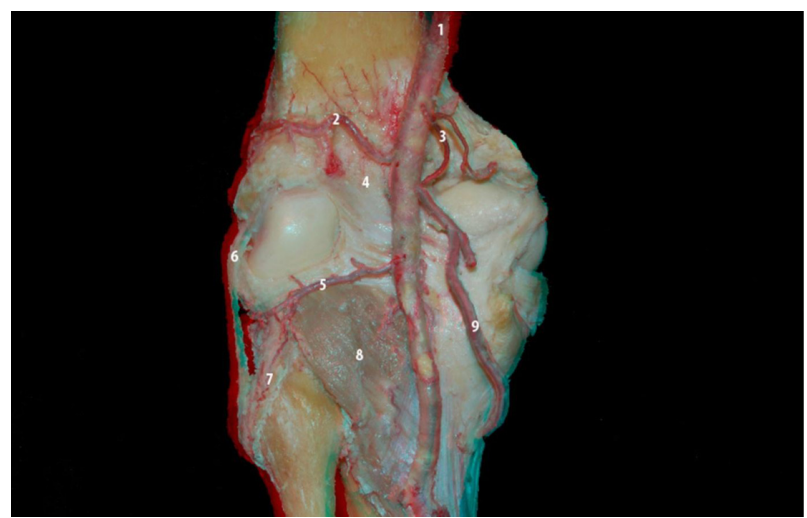

Figure 7 Posterior view of the knee: lateral inferior genicular artery and the posterolateral compartment structures: popliteal artery (I), lateral superior genicular artery (2), medial superior genicular artery (3), posterior septum of the knee (4), lateral inferior genicular artery (5), collateral lateral ligament (6), popliteofibular ligament (7), popliteal muscle (8), and medial inferior genicular artery (9).

of osteonecrosis, compared with the medial condyle. The 3D visualization can clearly convey the large number of vessels and their penetration in the subchondral bone (Figure 8).

An additional advantage is the ability to correlate the position of the same structures when the knee is in different positions. It is necessary to know the $2 \mathrm{D}$ anatomy of these structures to make a relationship with the $3 \mathrm{D}$ images. One example of this is the relationship between the proximal attachment of the popliteus tendon and the lateral collateral ligament where lateral images of the knee during extension and flexion are acquired.

It is helpful to divide this anatomical region into layered structures. In the first or external layer, there are the muscle groups of the iliotibial band, biceps femoris, and the lateral head of the gastrocnemius. ${ }^{12}$ The second layer or intermediate layer corresponds to the lateral collateral ligament,

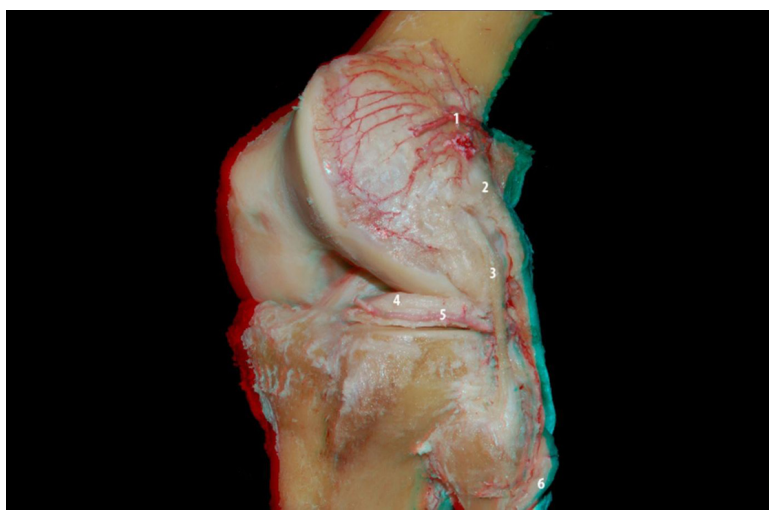

Figure 8 Oblique view of the posterolateral compartment of the knee: the pathway of the lateral inferior genicular artery (5) and the relationship with other structures: lateral superior genicular artery (I), popliteus tendon (2), lateral collateral ligament (3), lateral meniscus (4), and fibular nerve (6).

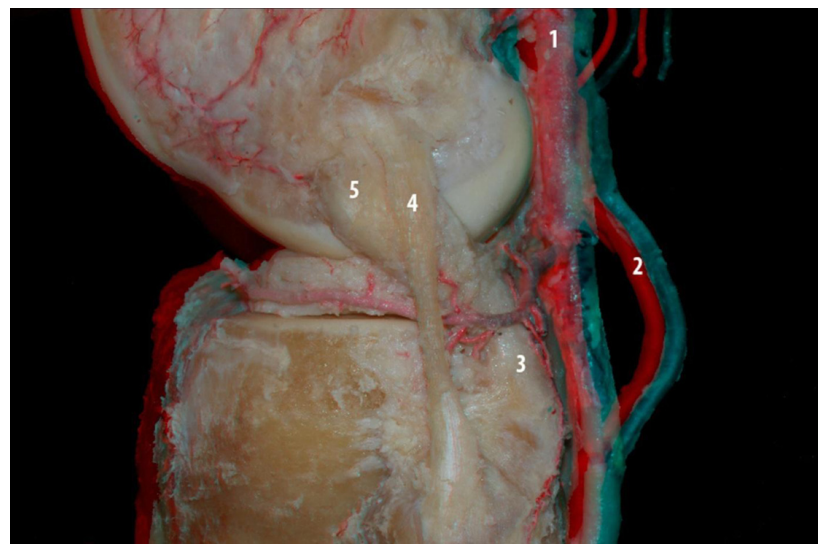

Figure 9 Lateral view of the posterolateral compartment of the knee: the pathway of the lateral inferior genicular artery and the relationship with other structures of the posterolateral compartment: popliteofibular ligament (3), lateral collateral ligament (4), and popliteus tendon (5). Popliteal artery (I) and inferior medial genicular artery (2).

popliteofibular ligament, and the popliteus tendon. The third layer or deep layer corresponds to the fabellofibular ligament, arcuate ligament, coronary ligament, and joint capsule. ${ }^{13-15}$

One limitation of the study was the limited number of dissected knees, not allowing the precise analysis of anatomical variance. Additionally, it was not possible to visualize the arcuate ligament and the fabellofibular ligament in the dissected knees, which along with the joint capsule would be the third layer of this compartment.

Different anatomical patterns were not found when compared with that shown by the literature. But the relationship between these structures can be understood much more clearly when viewed in 3D. The use of 3D technology to visualize the posterolateral corner of the knee helps improve the understanding of these structures and their relationships.

\section{Disclosure}

The authors report no conflicts of interest in this work.

\section{References}

1. Scapinelli R. Vascular anatomy of the human cruciate ligaments and surrounding structures. Clin Anat. 1997;10(3):151-162.

2. Park SE, Stamos BD, DeFrate LE, Gill TJ, Li G. The effect of posterior knee capsulotomy on posterior tibial translation during posterior cruciate ligament tibial inlay reconstruction. Am J Sports Med. 2004; 32(6):1514-1519.

3. Mariani PP, Margheritini F. Full arthroscopic inlay reconstruction of posterior cruciate ligament. Knee Surg Sports Traumatol Arthrosc. 2006; 14(11):1038-1044.

4. Bollen S. Epidemiology of knee injuries: diagnosis and triage. Br J Sports Med. 2000;34(3):227-228.

5. Sanchez AR 2nd, Sugalski MT, LaPrade RF. Anatomy and biomechanics of the lateral side of the knee. Sports Med Arthrosc. 2006;14(1):2-11.

6. Sekiya JK, Jacobson JA, Wojtys EM. Sonographic imaging of the posterolateral structures of the knee: findings in human cadavers. Arthroscopy. 2002;18(8):872-881. 
7. Arciero RA. Anatomic posterolateral corner knee reconstruction. Arthroscopy. 2005;21(9):1147.

8. Ribas GC, Bento RF, Rodrigues AJ Jr. Anaglyphic three-dimensional stereoscopic printing: revival of an old method for anatomical and surgical teaching and reporting. J Neurosurg. 2001;95(6):1057-1066.

9. Shimizu S, Tanaka R, Rhoton AL Jr, et al. Anatomic dissection and classic three-dimensional documentation: a unit of education for neurosurgical anatomy revisited. Neurosurgery. 2006;58(5):E1000.

10. Ferwerda JG. The World of 3-D: A Practical Guide to Stereo Photography. 2nd ed. Eeserveen, the Netherlands: 3-D Book Productions; 1990.

11. Cohen M, Costa Astur D, Kaleka CC, et al. Introducing 3-dimensional stereoscopic imaging to the study of musculoskeletal anatomy. Arthroscopy. 2011;27(4):593-596.
12. Vieira EL, Vieira EA, da Silva RT, Berlfein PA, Abdalla RJ, Cohen M. An anatomic study of the iliotibial tract. Arthroscopy. 2007; 23(3):269-274.

13. Seebacher JR, Inglis AE, Marshall JL, Warren RF. The structure of the posterolateral aspect of the knee. J Bone Joint Surg Am. 1982;64(4): 536-541.

14. LaPrade RF, Resig S, Wentorf F, Lewis JL. The effects of grade III posterolateral knee complex injuries on anterior cruciate ligament graft force. A biomechanical analysis. Am J Sports Med. 1999;27(4): 469-475.

15. LaPrade RF, Muench C, Wentorf F, Lewis JL. The effect of injury to the posterolateral structures of the knee on force in a posterior cruciate ligament graft: a biomechanical study. Am J Sports Med. 2002;30(2): $233-238$.

\section{Publish your work in this journal}

Open Access Journal of Sports Medicine is an international, peer-reviewed, open access journal publishing original research, reports, reviews and commentaries on all areas of sports medicine. The manuscript management system is completely online and includes a very quick and fair peer-review system.
Visit http://www.dovepress.com/testimonials.php to read real quotes from published authors. 